for the interpretation of the radio data. On the other hand, barred spirals often show nearly circular rings superposed on a bar, e.g., NGC 4725 , or branches that bend sharply from the ends of the bar, e.g., NGC I300. The optical arms of the Galaxy are observed as segments too short by a factor of 2 or 3 for a good check as to whether they represent branches near the end of a bar whose axis slightly precedes the sun.

The second observation is of the neutral hydrogen about the direction of the galactic center (Kwee, Muller, and Westerhout 1954). The Leiden group proposes a central disk of mostly ionized hydrogen that extends $3 \mathrm{kpc}$ from the center in highly turbulent motion. This is surely not like the M 3 I nucleus, which is free of $H \alpha$ emission and which shows spiral arms that become finer, not more turbulent-looking, as they enter it. The disk does not explain asymmetries in the $2 \mathrm{I}-\mathrm{cm}$ line profiles, such as the wings of positive and negative velocity over $32 \mathrm{1}^{\circ} \leq l<329^{\circ}$ and the single positive wing of greater intensity over $329^{\circ}<l<343^{\circ}$. However, a slightly inclined and foreshortened bar would be observed over unsymmetrical parts and distances on either side of center, perhaps as described.

The third observation is of longitudinal asymmetries of the continuous radio radiation in the Galaxy (Pawsey and Bracewell 1955). Isophotes for a wide range of frequencies show that the preceding hemisphere (pole $l=55^{\circ}, b=0^{\circ}$ ) radiates more than the following hemisphere at all latitudes. Some particulars of this imbalance are the north and south bulges roughly along the circle $l=350^{\circ}$ and the saddle minimum near $l=200^{\circ}, b=0^{\circ}$, or $50^{\circ}$ past the anti-center. Spiral arms and an axially symmetric disk fail to explain these asymmetries, but a radiating bar may suit them if the sun lies within its trailing edge.

Kwee, K. K., Muller, C. A. and Westerhout, G. I954, B. A. N., No. $45^{8}$.

Pawsey, J. L. and Bracewell, R. N. 1955, Radio Astronomy, (Oxford: at the Clarendon Press), Chap. 7.

State University of Iowa, Iowa City, Iowa.

\section{Kameny, Franklin E. Photoelectric colors of RV Tauri and yellow semiregular variables.}

Three-color photoelectric observations, tied to the UBV system of Johnson, were made of the RV Tauri-type and yellow semiregular variables AG Aur, TW Cam, SS and SU Gem, AB Leo, U Mon, RV Tau, and SV UMa. All were observed through at least one complete cycle. Less complete observations were made of UY and UZ CMa, TX Per, WW Tau, and V Vul.

Intrinsic colors, compared with those of Johnson's standard supergiants, show the RV Tauri stars are all too blue for their spectral classes, while the yellow semiregular variables are all too red. The latter stars have $(U-B)$ colors too early for their $(V-B)$ colors, indicating an ultraviolet excess.

It appears that all these stars reach maximum radius about halfway down the descending branch of the light curve. On the average, and with considerable uncertainty, $r_{\max }$ is about $1.54 r_{\min }$ for the RV Tauri stars, and about $1.40 r_{\min }$ for the yellow semiregular variables. Mean radii, derived with the aid of published radial velocities, are of the order of $100 \times 10^{6} \mathrm{~km}$, leading to visual absolute magnitudes about -5.3 for the RV Tauri stars, and about -4.4 for the yellow semiregular variables.

Harvard College Observatory, Cambridge, Mass.

\section{Kapany, N. S. A light funnel for stellar spectro- graph.}

A new method using "static scanning" is described to funnel the light flux contained in the star image into the spectrograph slit. Use is made of fine glass fibers to conduct light from one point to another. One end of the fiber bundle is clamped in the shape required of the secondary source, the spectrograph slit, and the other end is clamped into a shape similar to that of the primary source, the star disc.

Methods for fabricating such light funnels will be described. Laboratory measurements on the photometric efficiency of certain working models indicate that a considerable increase in efficiency seems possible by this method.

Optical arrangements to insure uniform illumination at the slit and also to obviate the effects due to motion of the stellar image are suggested.

\section{Institute of Optics, University of Rochester, Rochester, $N$. $Y$.}

\section{King, Robert B. Status of absolute $f$-values for lines in spectra of the iron group.}

Absolute $f$-values have now been measured by different methods for lines in the spectra of several of the elements in the iron group. New vapor pressure data for some elements has permitted revision of $f$ values determined earlier from meas- 